\title{
Using 3D Printing Technology for Corrective Biplanar Chevron Osteotomy with Customized Osteotomy Guide and Patient-Matched Monoblock Crosslink Plate in Treatment of Cubitus Varus Deformity: A Case Report and Technical Note
}

Nithid Sri-utenchai

Mahidol University Faculty of Medicine Ramathibodi Hospital

Nachapan Pengrung

Mahidol University Faculty of Medicine Ramathibodi Hospital

Korakod SrikonKorn

Chulalongkorn University

Chedtha Puncreobutr

Chulalongkorn University Faculty of Engineering

Boonrat Lohwongwatana

Chulalongkorn University Faculty of Engineering

Paphon Sa-ngasoongsong ( $\square$ paphonortho@gmail.com )

Mahidol University Faculty of Medicine Ramathibodi Hospital https://orcid.org/0000-0003-1936-4456

\section{Technical note}

Keywords: cubitus varus deformity, 3D printing technology, biplanar Chevron osteotomy, customized osteotomy guide, patient-matched implant, monoblock crosslink plate

Posted Date: August 19th, 2020

DOI: https://doi.org/10.21203/rs.3.rs-61558/v1

License: (c) (i) This work is licensed under a Creative Commons Attribution 4.0 International License.

Read Full License 


\section{Abstract \\ Background}

Cubitus varus deformity (CVD), a common complication after a supracondylar fracture of the distal humerus, is usually treated with corrective osteotomy. However, due to the complex anatomy of the distal humerus, conventional osteotomy techniques are sometimes unreliable and can result in an inaccurate correction, residual deformity, and lateral condyle prominence. Recently, medial three-dimensional (3D) printing technology has demonstrated potential benefits for the treatment of CVD by improving the accuracy of the osteotomy through the use of an osteotomy guide with or without a patient-mated plate. This study aimed to present an interesting CVD case involving a patient who was treated with corrective biplanar Chevron osteotomy using an innovative customized osteotomy guide and a newly designed patient-matched monoblock crosslink plate created with 3D printing technology.

\section{Methods}

A computer simulation was processed using images from computerized tomography(CT) scans of both upper extremities. The biplanar Chevron osteotomy was designed to create identical anatomy between the mirror image of the contralateral distal humerus and the osteotomized distal humerus. Next, the customized osteotomy guide and patient-matched monoblock crosslink plate were designed and printed. A simulation osteotomy for the real-sized bone model was created.

\section{Results}

The operation was performed using the posterior paratricipital approach and the k-wire position from the customized osteotomy guide as a predrilled hole for screw fixation to achieve immediate control of the reduction after osteotomy. Our method helped successfully treat the CVD in the case study patient and significantly improved her radiographic and clinical outcomes with a satisfactory result.

\section{Conclusion}

This study showed that the treatment of CVD using 3D printing technology to create an innovative customized osteotomy guide and a patient-matched monoblock crosslink plate can help accurately assess and control the CVD correction. To the best of our knowledge, this case report introduces a new insight for the clinical application of 3D printing technology in the treatment of CVD.

\section{Background}

Cubitus varus deformity (CVD) is the most common late complication after supracondylar fracture of distal humerus in children with a reported incidence of up to $50 \%$ [1]. The etiologies of this deformity 
derive mostly from malunion and rarely from avascular necrosis with or without growth arrest of the medial physis [2], which results in multiplanar deformity (varus, hyperextension, and rotation) [3]. Current standard treatment of CVD is corrective supracondylar osteotomy of the humerus, and many surgical correction techniques have been described in the literature [2]. However, previous studies showed that the conventional corrective osteotomy can result in poor functional outcomes-such as unsatisfactory correction and residual deformity, especially in horizontal and rotational planes-and lateral condyle prominence with poor cosmetic appearance $[4,5]$.

Recently, three-dimensional (3D) printing technology has been continually developing and become one of the most exciting and fastest growing fields in healthcare due to excellent accuracy that stems from using preoperative computerized tomography (CT) evaluations and advanced computer software; this technology enables personalized treatment and introduces wide-ranging potential uses, including in the clinical application of orthopedic surgery [6]. Regarding the treatment of CVD, previous studies showed that 3D printing technology is beneficial for preoperative planning [7], designing individualized surgical guides $[8,9,10]$, preparing prebent plates with real-sized plastic bone models [11], and printing patientmatched plates $[12,13]$. However, to the best of our knowledge, the application of 3D printing technology in the treatment of CVD remains limited to designing osteotomy guides for simple techniques, such as lateral closed-wedge osteotomy, and mostly fixations with conventional implants. Therefore, this study aims to present the case report of a 32-year-old female with CVD who has been treated with corrective biplanar Chevron osteotomy using a customized osteotomy guide and a patient-matched monoblock crosslink plate.

\section{Materials And Methods}

\section{Case report}

A 32-year-old Thai female presented with left elbow deformity for 24 years. She had a history of injury, including a fall from a chair at age 6 that resulted in being treated with a conservative method by a traditional doctor. Afterward, she noticed a progressive gunstock deformity of her left elbow without pain, numbness, or weakness in her left upper extremities. However, per patient report, due to the appearance of the deformity, she lacked confidence and did not wear sleeveless shirts or vests. Physical examination of left elbow showed a $20^{\circ}$ varus deformity with elbow range of motion (ROM) as flexion of $125^{\circ}$, extension of $-10^{\circ}$, supination of $85^{\circ}$, and pronation of $80^{\circ}$ (Fig. 1). There was no muscle atrophy of the left hand, and the distal neurovascular status of the left upper extremity was intact. The preoperative radiographs (Fig. 2) and 3D CT analysis (Fig. 3) showed a $15^{\circ}$ varus deformity and $10^{\circ}$ hyperextension deformity without rotational deformity. Therefore, she was diagnosed as having a cubitus varus and recurvatum deformity of the left distal humerus.

The treatment option for her deformity was discussed. The planned operation was a corrective biplanar Chevron osteotomy with a customized osteotomy guide and an innovative patient-matched monoblock crosslink titanium plate (Fig. 4). In this case, we decided to use the 3D printing technology to create the 
customized osteotomy guide and the patient-matched plate due to the effective accuracy of this technology for osteotomy compared to the conventional technique and due to the better plate profile on the patient's anatomy. The customized osteotomy guide was specifically designed for the position of kwire fixation at the same position of the screw holes on the patient-matched plate. Through these aspects, this specific design can assist the surgeon with bone reduction after osteotomy via application of the plate through the k-wire position, which has the predrilled hole for screw insertion. Moreover, we also designed the plate configuration as a monoblock crosslink plate, by placing the plate on the medial and posterolateral side of the distal humerus with the built-in metal crosslink between both sides. This plate design would be helpful for the reduction osteotomy due to the easier and better intraoperative plate position adjustment, compared to the conventional dual plating technique. We also felt the crosslink would improve the biomechanical property of the plate and the stability after fixation compared to the separate dual plates, just like in spinal surgery [14]. Therefore, using both the innovative plate design with these combined advantages and the 3D printing technology would be helpful for the complex osteotomy operation by reducing the operative time related to the bone reduction step and improving the accuracy of the osteotomy.

\section{Preparation of customized osteotomy guide and patient-matched monoblock crosslink plate}

To fabricate the patient-matched plate and customized osteotomy guide, a simulation of the deformity correction was done through 3D reconstruction and image processing of DICOM files, taken from CT scans, via Avizo software (Thermo Fisher Scientific, MA, USA). A corrective biplanar Chevron osteotomy was planned, as shown in Fig. 4A, to divide the affected bone into 3 separated fragments (Fig. 4B, C). The distal fragment of bone (dark blue) was then reduced to match the mirrored image of the contralateral bone (green), as displayed in Fig. 4D. The design of the customized osteotomy guide and the patientmatched monoblock crosslink plate were then developed based on anatomical bone geometry and the surgeon's requirements using ANSYS software (Ansys Inc., PA, USA).

The osteotomy guide was designed to fit over the distal surfaces (posterior, lateral, and medial supracondylar ridge) of the defected bone, except the area over the olecranon fossa (Fig. 4E, F). The cutting slots and drilling sleeves were designed to match the cutting planes and the plate's screw holes, respectively. The patient-specific plate with the monoblock crosslink was designed to fit over the corrected humerus bone with an overall thickness of $2 \mathrm{~mm}$ (Fig. 4G, H). The structural strength of the plate was also confirmed by finite element analysis.

Upon the surgeon's approval of the design, the patient-specific implant was 3D-printed by selective laser melting using medical grade Ti-6Al-4V alloy (Meticuly Co., Ltd., Thailand). Subsequent post-processing techniques, including surface polishing and sterilization, were performed according to routine standards. For osteotomy guides and bone models, fused filament fabrication was used for the 3D printing of biocompatible high-impact polystyrene. The entire fabrication process was certified by the IS013485 standard for the design, manufacturing, and sterilization of medical devices. Finally, the customized 
osteotomy guide was printed, and the trial osteotomy was simulated on the real-sized plastic bone model (Fig. 5).

\section{Surgical technique and postoperative care}

The operation, a corrective biplanar Chevron osteotomy of the distal humerus, was performed by the orthopedic trauma expert (PS), who has more than 10 years of experience in orthopedic reconstruction surgery. The standard posterior paratricipital surgical approach was used. After combined general anesthesia and an ultrasound-guided supraclavicular nerve block, the patient was placed in the right lateral decubitus position. A $20-\mathrm{cm}$ posterior midline incision was made (Fig. 6A). The triceps muscle was exposed, and then the ulnar nerve was identified. The muscle was elevated from the posterior surface of the distal humerus, and the customized osteotomy guide was placed and fixed with multiple K-wires (Fig. 6B). After complete osteotomy, the guide and bone blocks were removed (Fig. 6C), and the plate was inserted under the triceps using the screw fixation based on the predrilled screw holes (Fig. 6D). The intraoperative flexion/extension ROM was $135^{\circ} / 0^{\circ}$. The intraoperative fluoroscopic images were checked to confirm the post-reduction alignment (Fig. 6E) before wound closure without nerve transposition. The total operative time was 116 minutes. The initial postoperative radiographs showed good alignment and adequate fixation stability in both AP and lateral views (Fig. 6F).

After surgery, the routine postoperative care was pain control with multimodal analgesia and the application of intermittent cold compressions. Antibiotic prophylaxis was given for 24 hours. The patient was immobilized with an arm sling for 6 weeks and was allowed to perform active assisted-ROM exercises of the wrist, elbow, and shoulder joints as tolerated. During admission, she reported only minimal pain on motion with mild paresthesia on the ulnar side of the left hand. She was discharged from the hospital 3 days postoperatively and was then scheduled for follow-up visits at the orthopedic clinic. On the follow-up, the clinical examination showed nearly normal alignment of the left elbow compared to the right side. The ROM for flexion/extension and pronation/supination of the left elbow were $130^{\circ} / 0^{\circ}$ and $90^{\circ} / 90^{\circ}$, respectively (the right elbow ROM was $130^{\circ} / 0^{\circ}$ and $85^{\circ} / 90^{\circ}$, respectively). The normal sensation had completely returned at 6 weeks, and the osteotomy had healed uneventfully at 3 months postoperatively. The postoperative radiographs demonstrated the improved humerus-elbowwrist angle from $15^{\circ}$ varus to $7^{\circ}$ valgus (compared to $8^{\circ}$ valgus on the right side) (Fig. 7). Through the last follow-up visit, at 18 months postoperatively, she reported being highly satisfied with the clinical outcome and did not report feeling any pain, swelling, or implant irritation. Therefore, we did not advise to remove the implant in this case.

Patient has given informed consent for data allowance and publication. The present study has been reviewed and approved by the Institutional Review Board at Mahidol University, based on the Declaration of Helsinki.

\section{Discussion}


Surgical correction of the CVD is one of the most challenging orthopedic reconstruction surgical procedures due to the need for comprehensive analysis in the distorted anatomy in all planes, including varus deformity in the coronal plane, hyperextension deformity in the sagittal plane, and internal rotation deformity in the horizontal plane [3]. Achieving a successful outcome usually requires both an accurate method for measuring the deformity and a precise surgical method for corrective osteotomy. Therefore, medical 3D printing technology has fueled new hope for an effective treatment of the CVD. The technology employs 3D reconstruction based on the CT scanning data and uses computer software and rapid prototype technology to create a preoperative template, customized osteotomy guide, and patientmatched implant. Especially promising is that the technology enables highly accurate control of the plane and the angle of the osteotomy, resulting in higher overall operating accuracy and a lower risk of complications.

Regarding the development of a customized osteotomy guide for the CVD, Murase et al. first reported performing a 3D corrective osteotomy on 22 patients with malunited fractures of the upper extremities (including 4 cases with CVD) by using the custom-made osteotomy template and the reduction guide. The custom-made osteotomy was designed to be placed on the bone surface of the lateral side of the distal humerus metaphysis and fixated with k-wire. Then, the closed-wedge osteotomy was performed by cutting through a slit on the template and using the reduction guide to align the bone [15]. Since then, many researchers have tried to develop an individualized navigation template for the lateral closed-wedge osteotomy for the CVD $[9,16,17]$ and showed the advantages of this technique with respect to determining the osteotomy angle, osteotomy plane, and rotational angle. In this study, we innovatively designed the customized osteotomy guide to place on the posterior surface of the distal humerus due to the need for a posterior slit for the biplanar Chevron osteotomy. Although this design might be larger than the previously mentioned lateral-side template, our customized osteotomy guide provides predrilled holes for medial plate placement, resulting in easier placement of the 3D patient-matched plate in the later steps. Moreover, the osteotomy through a posterior slit would better minimize the risk of ulnar nerve injury compared to an osteotomy through a lateral slit.

Concerning the development of the patient-matched plate for the CVD, there were only two studies-one by Omori et al. [12] in a cadaveric study and the other by Oka et al. [13] in a prospective multicenter study. In these studies, both patient-matched plate designs were made from titanium and placed on the lateral side of the distal humerus. Although there was no control group in the prospective study by Oka et al, they showed that the patient-matched implants resulted in highly accurate correction with an average residual maximal deformity angle of $3.3^{\circ}$ and an average $3 \mathrm{D}$ correction error of $<1.0^{\circ}$ and $<1 \mathrm{~mm}$, with high patient satisfaction and no serious complications.

Our study also has some limitations. First, although the present study has demonstrated a successful outcome, there was only one case in our report and no control group. Therefore, the result of this study cannot directly compare with the techniques in the previous literature. In addition, our results did not explore the actual benefits of the crosslink design. However, we believe our method is reliable; like the methods in the previous studies, our method has high accuracy as well. Second, due to the osteotomy 
and implant design with placement on both the medial and posterolateral surface of the distal humerus, this method requires the posterior paratricipital approach to provide for a medial and lateral soft tissue window and elevation of the triceps muscle. However, this posterior approach allows the surgeon to explore and protect the ulnar nerve, which is safer for patients with the type of severe and complex anatomy present with CVD. Moreover, the surgical scar on the posterior side resulting from this approach is more cosmetically acceptable for patients, especially for younger females. Lastly, as with previous studies using 3D printing technology, this method requires patients to be exposed to radiation from the CT required for both upper extremities.

\section{Conclusion}

This study showed that using 3D printing technology to assist with a customized osteotomy guide and patient-matched monoblock crosslink plate is an interesting option for the treatment of CVD. The method was found to provide accurate control of the deformity correction, avoid a prolonged operative time or unnecessary blood loss, and decrease the risk of postoperative complications.

\section{Declarations}

\section{Availability of data and materials}

The data used to support the findings of this study are available from the corresponding author upon request.

\section{Competing interests}

All authors declare that there is no conflict of interest regarding the publication of this article.

\section{Funding statement}

This work was supported by the Biomedical Engineering Research Center, Chulalongkorn University and the Faculty of Medicine Ramathibodi Hospital, Mahidol University. No external funding was obtained for this study.

\section{Authors' contributions}

All authors helped conceptualize and design the study. NS and NP collected the data and prepared the manuscript. PS designed the customized osteotomy guide and the patient-matched plate, performed the operation, and helped to prepare and revise the manuscript. KS performed computer simulation. KS, CP, and $\mathrm{BL}$ all helped manufacture the 3D printing design. $\mathrm{CP}$ and $\mathrm{BL}$ helped to critically revise the manuscript to ensure it contained highly intellectual content. All authors read and approved the final manuscript.

\section{Acknowledgments}


The authors would like to thank the Department of Orthopedics, Faculty of Medicine Ramathibodi Hospital, Mahidol University and the Biomedical Engineering Research Center and Department of Metallurgy Engineering, Faculty of Engineering, Chulalongkorn University for all of the kind assistance and for permission to conduct the study.

\section{References}

1. Tellisi N, Abusetta G, Day M, Hamid A, Ashammakhi N, Wahab KH. Management of Gartland's type III supracondylar fractures of the humerus in children: the role audit and practice guidelines. Injury. 2004 Nov;35(11):1167-71. PubMed PMID: 15488510. Epub 2004/10/19.

2. Verka PS, Kejariwal U, Singh B. Management of cubitus varus deformity in children by closed dome osteotomy. J Clin Diagn Res. 2017 Mar;11(3):RC08-RC12. PubMed PMID: 28511466. Pubmed Central PMCID: PMC5427392. Epub 2017/05/18.

3. Takeyasu Y, Murase T, Miyake J, Oka K, Arimitsu S, Moritomo H, et al. Three-dimensional analysis of cubitus varus deformity after supracondylar fractures of the humerus. J Shoulder Elbow Surg. 2011 Apr;20(3):440-8. PubMed PMID: 21397793. Epub 2011/03/15.

4. Kim HT, Lee JS, Yoo Cl. Management of cubitus varus and valgus. J Bone Joint Surg Am. 2005 Apr;87(4):771-80. PubMed PMID: 15805206. Epub 2005/04/05.

5. Cho CH, Song KS, Min BW, Bae KC, Lee KJ. Long-term results of remodeling of lateral condylar prominence after lateral closed-wedge osteotomy for cubitus varus. J Shoulder Elbow Surg. 2009 May-Jun;18(3):478-83. PubMed PMID: 19393939. Epub 2009/04/28.

6. Aimar A, Palermo A, Innocenti B. The role of 3D printing in medical applications: a state of the art. J Healthc Eng. 2019;2019:5340616. PubMed PMID: 31019667. Pubmed Central PMCID: PMC6451800. Epub 2019/04/26.

7. Bovid KM, Kohler EJ, Habeck JM, Gustafson PA. Utilization of a 3D-printed model for preoperative planning and operative osteotomy of a pediatric cubitus varus deformity. JSES Open Access. 2019 Oct;3(3):219-24. PubMed PMID: 31709366. Pubmed Central PMCID: PMC6835026. Epub 2019/11/12.

8. Murase T, Takeyasu Y, Oka K, Kataoka T, Tanaka H, Yoshikawa H. Three-dimensional corrective osteotomy for cubitus varus deformity with use of custom-made surgical guides. JBJS Essent Surg Tech. 2014 Mar;4(1):e6. PubMed PMID: 30775113. Pubmed Central PMCID: PMC6355040. Epub 2014/03/26.

9. Vlachopoulos L, Schweizer A, Meyer DC, Gerber C, FurnstahI P. Three-dimensional corrective osteotomies of complex malunited humeral fractures using patient-specific guides. J Shoulder Elbow Surg. 2016 Dec;25(12):2040-7. PubMed PMID: 27503533. Epub 2016/08/10.

10. Zhang YZ, Lu S, Chen B, Zhao JM, Liu R, Pei GX. Application of computer-aided design osteotomy template for treatment of cubitus varus deformity in teenagers: a pilot study. J Shoulder Elbow Surg. 2011 Jan;20(1):51-6. PubMed PMID: 21134665. Epub 2010/12/08. 
11. Kataoka T, Oka K, Miyake J, Omori S, Tanaka H, Murase T. 3-Dimensional prebent plate fixation in corrective osteotomy of malunited upper extremity fractures using a real-sized plastic bone model prepared by preoperative computer simulation. J Hand Surg Am. 2013 May;38(5):909-19. PubMed PMID: 23566719. Epub 2013/04/10.

12. Omori S, Murase T, Kataoka T, Kawanishi Y, Oura K, Miyake J, et al. Three-dimensional corrective osteotomy using a patient-specific osteotomy guide and bone plate based on a computer simulation system: accuracy analysis in a cadaver study. Int J Med Robot. 2014 Jun;10(2):196-202. PubMed PMID: 24106094. Epub 2013/10/10.

13. Oka K, Tanaka H, Okada K, Sahara W, Myoui A, Yamada T, et al. Three-dimensional corrective osteotomy for malunited fractures of the upper extremity using patient-matched instruments: a prospective, multicenter, open-label, single-arm trial. J Bone Joint Surg Am. 2019 Apr 17;101(8):71021. PubMed PMID: 30994589. Epub 2019/04/18.

14. Brodke DS, Bachus KN, Mohr RA, Nguyen BK. Segmental pedicle screw fixation or cross-links in multilevel lumbar constructs. a biomechanical analysis. Spine J. 2001 Sep-Oct;1(5):373-9. PubMed PMID: 14588318. Epub 2003/11/01.

15. Murase T, Oka K, Moritomo H, Goto A, Yoshikawa H, Sugamoto K. Three-dimensional corrective osteotomy of malunited fractures of the upper extremity with use of a computer simulation system. J Bone Joint Surg Am. 2008 Nov;90(11):2375-89. PubMed PMID: 18978406. Epub 2008/11/04.

16. Hu X, Zhong M, Lou Y, Xu P, Jiang B, Mao F, et al. Clinical application of individualized 3D-printed navigation template to children with cubitus varus deformity. J Orthop Surg Res. 2020 Mar 19;15(1):111. PubMed PMID: 32192482. Pubmed Central PMCID: PMC7081535. Epub 2020/03/21.

17. Zhang YW, Xiao X, Gao WC, Xiao Y, Zhang SL, Ni WY, et al. Efficacy evaluation of three-dimensional printing assisted osteotomy guide plate in accurate osteotomy of adolescent cubitus varus deformity. J Orthop Surg Res. 2019 Nov 9;14(1):353. PubMed PMID: 31706346. Pubmed Central PMCID: PMC6842546. Epub 2019/11/11.

\section{Figures}



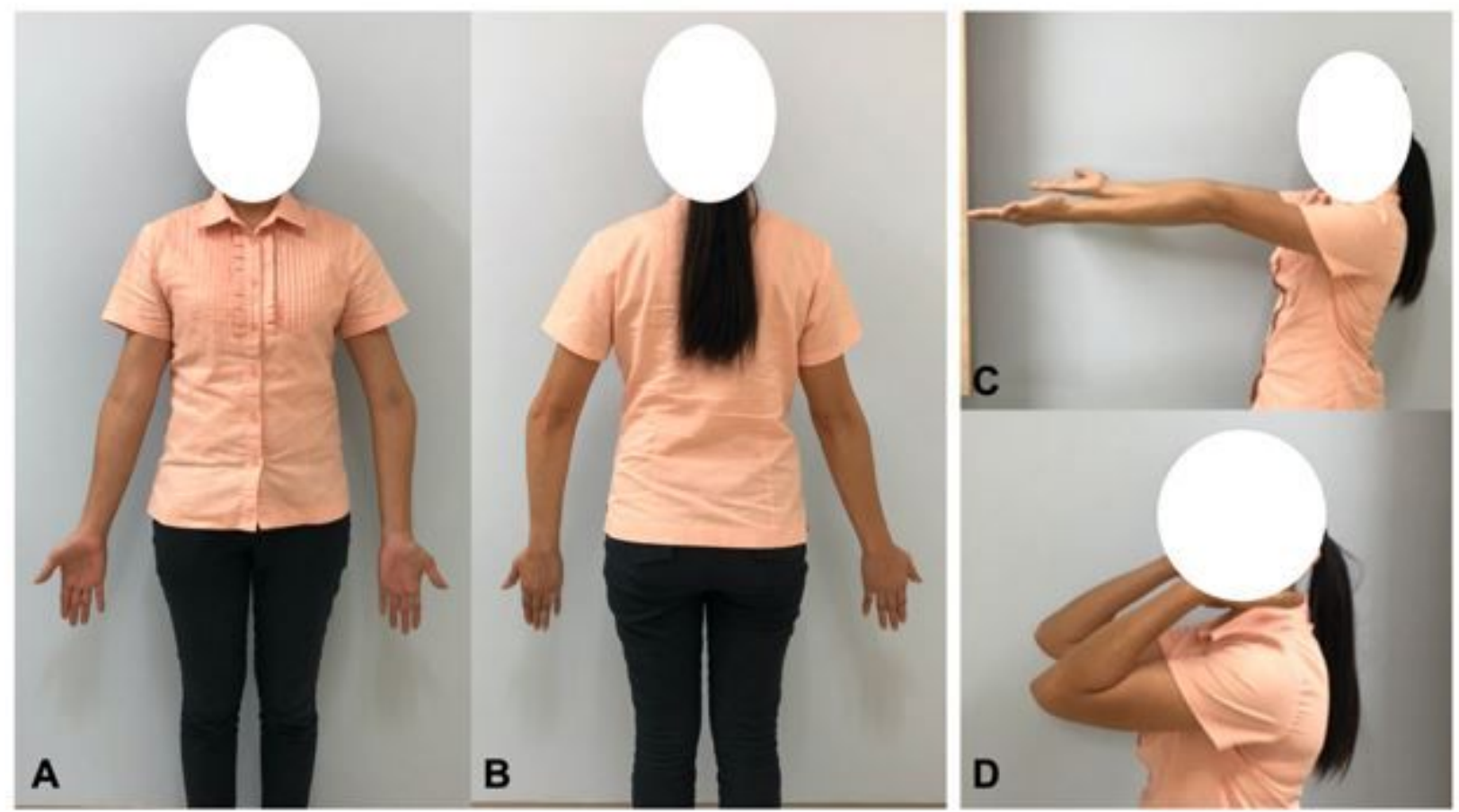

Figure 1

Clinical pictures showed the cubitus varus deformity ( $A$ and $B$ ) and hyperextension ( $C$ and $D)$ of the left elbow in a 32-year-old female.
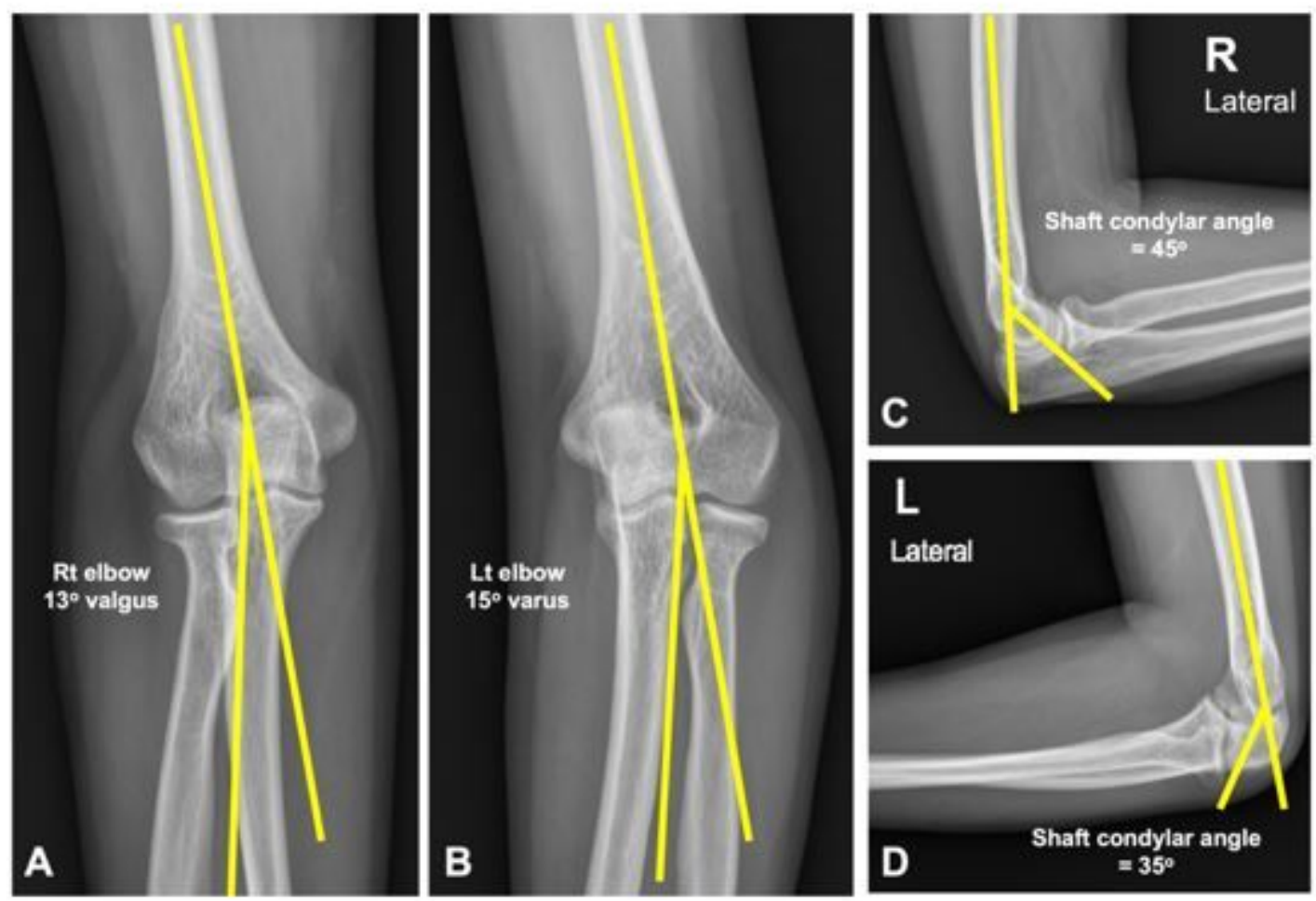

Figure 2 
Preoperative radiographs from both elbows. AP view of right (A) and left (B) elbows showed humeruselbow-wrist angle of 130 valgus and 150 varus, respectively. Lateral view of the right (C) and left (D) elbows demonstrated shaft condylar angles of 450 and 350 , respectively.

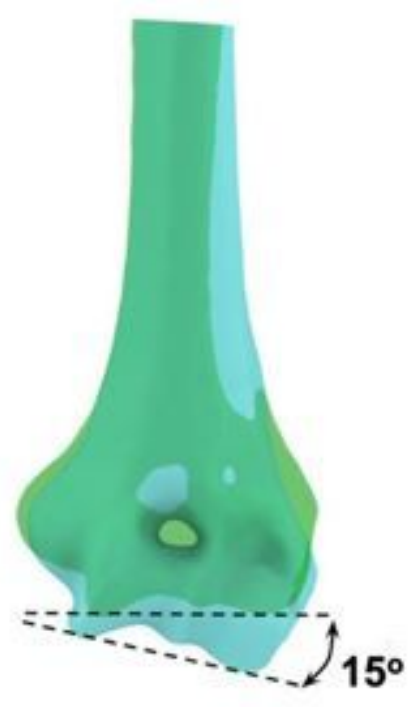

A

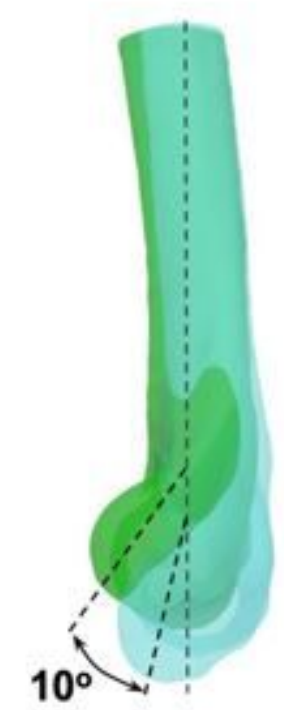

B

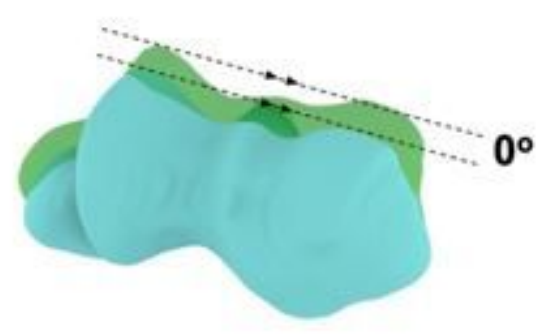

C

\section{Figure 3}

3D CT analysis with overlapping of the mirror image model from the right humerus (green color) and the deformed model from the left humerus (blue color). Anterior view shows a 15-degree varus deformity (A), lateral view shows a 10-degree hyperextension deformity (B), and axial view shows no rotational deformity (C). 

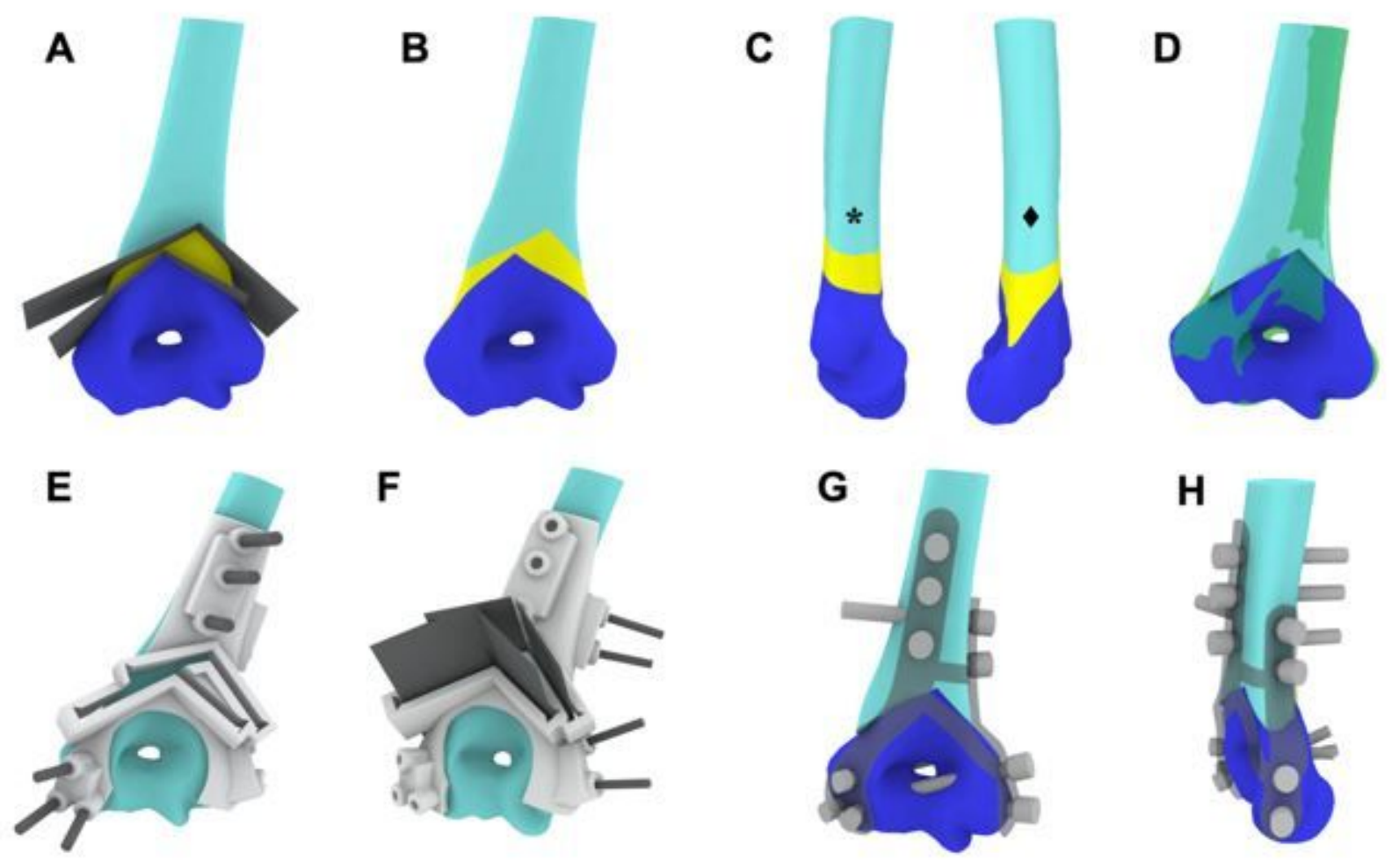

\section{Figure 4}

Preparation of the customized osteotomy guide and patient-matched plate. The biplanar Chevron osteotomy was designed $(\mathrm{A})$, and the osteotomy block in AP (B) and lateral views from medial ${ }^{*}$ ) and lateral $(\cdot)$ were demonstrated. The reduced bone model after osteotomy (dark blue color for the distal fragment and light blue color for the proximal fragment) was matched with the mirror model from the right humerus (green color). The customized osteotomy guide $(E-F)$ and the patient-matched monoblock crosslink plate $(\mathrm{G}-\mathrm{H})$ were made by using the same holes for $\mathrm{k}$-wire and screw fixation. 

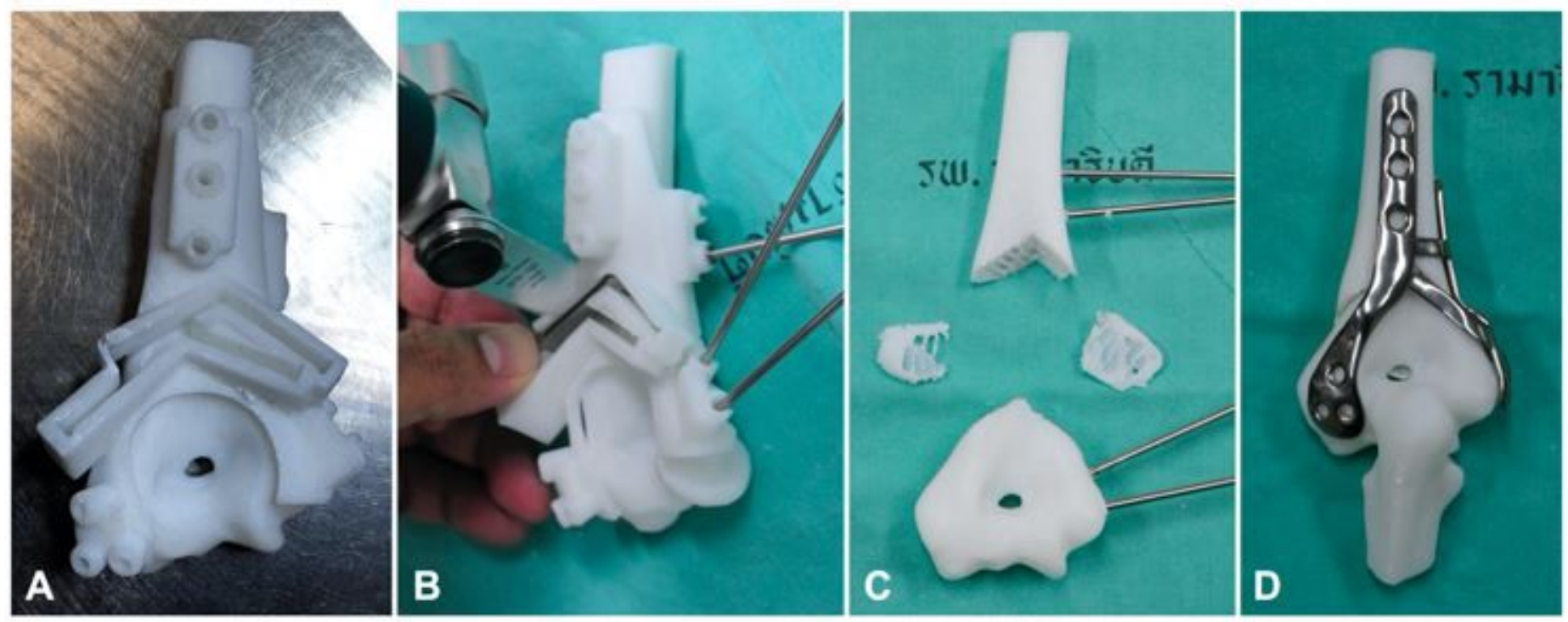

\section{Figure 5}

Simulation osteotomy. The customized osteotomy guide after placement on the real-sized plastic bone model (A), the model after being cut following the fixation of the customized osteotomy guide with k-wire (B), the separated bone model fragment after simulation osteotomy (C), and the bone model after reduction and the application of the patient-matched monoblock crosslink plate (D). 

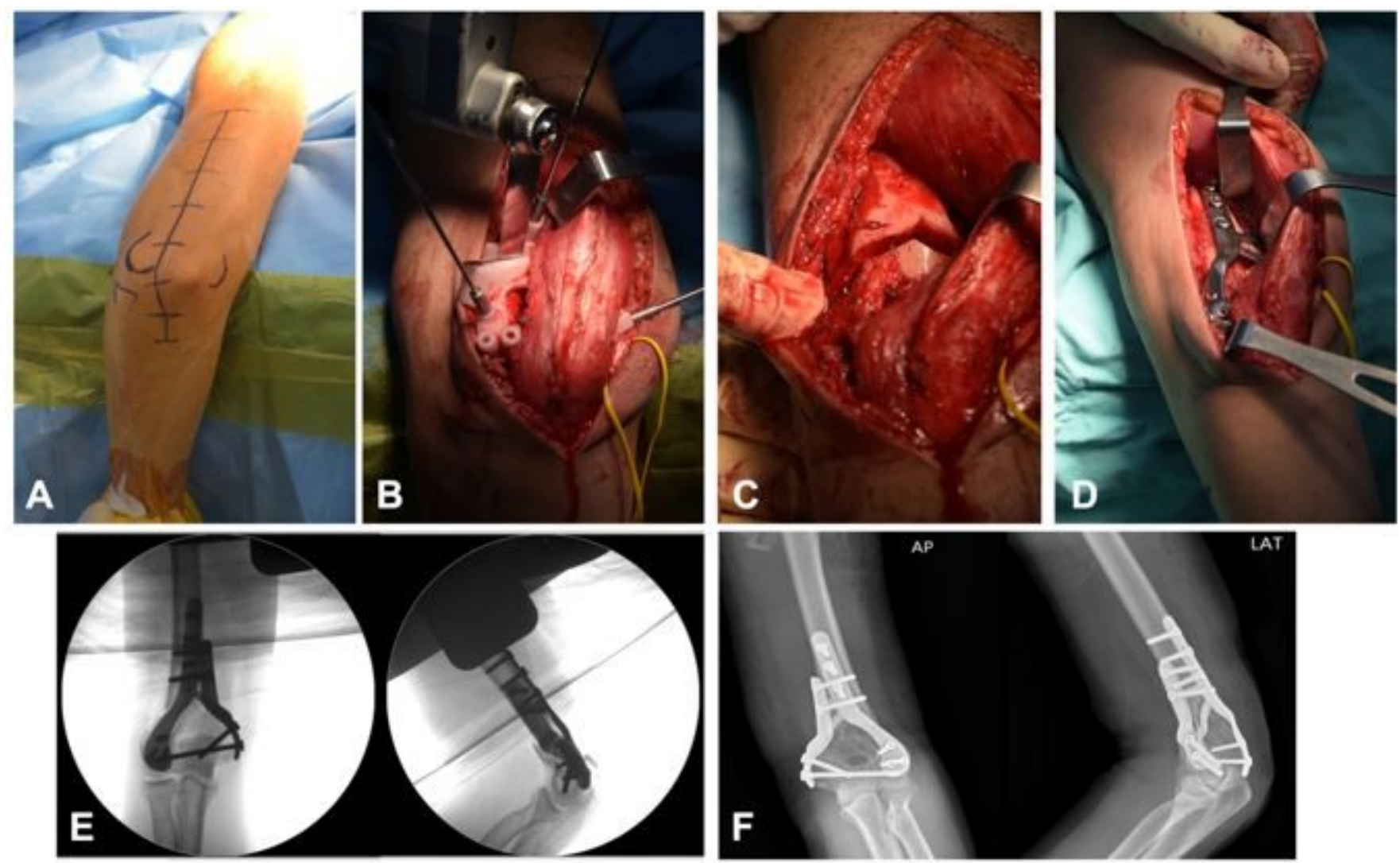

Figure 6

Intraoperative surgical techniques with intraoperative and initial postoperative images. The surgical method was the posterior approach in the lateral decubitus position (A). The customized osteotomy guide was placed and fixed after elevation of the triceps muscle and the identification of the ulnar nerve (yellow vessel loops) (B). The osteotomy was performed, and the bone blocks were removed (C). 

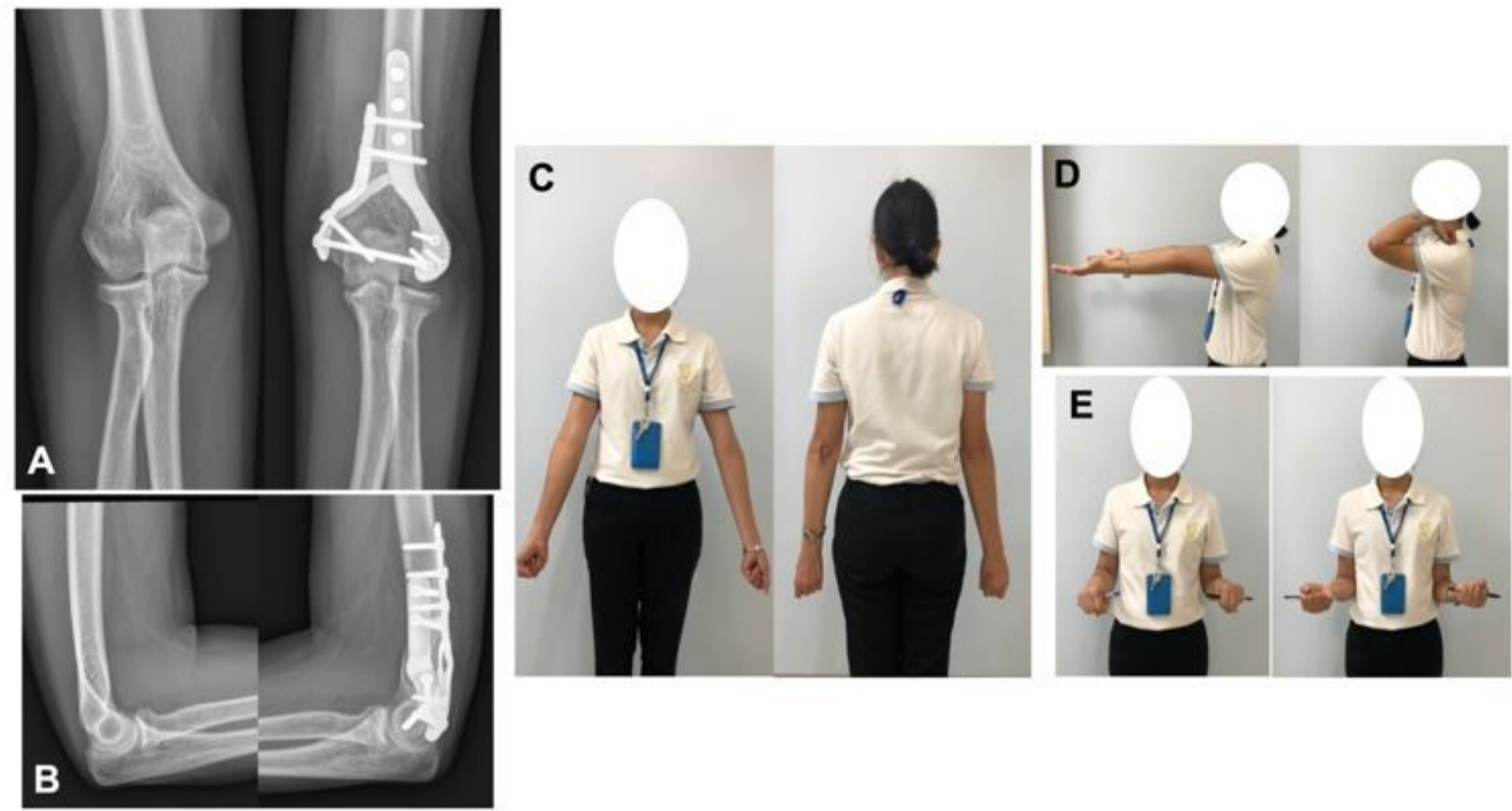

\section{Figure 7}

Postoperative radiographic and clinical outcomes. The 3-month postoperative radiographs of both elbows in AP (A) and lateral (B) views showed nearly anatomical alignment of the left elbow compared with the right elbow. The 3-month postoperative clinical examination revealed the nearly identical alignment of both elbows (C) with the same flexion/extension (D) and pronation/supination (E) ROM. 